\title{
GUIDELINES
}

\section{Chapter 12. Diagnosis of CKD in adults}

(C) Japanese Society of Nephrology 2009

- Many patients with adult CKD have chronic glomerulonephritis or diabetic nephropathy.

- CKD patients, if left untreated, have a risk of progressing in CKD stage.

- Polycystic kidney disease and gouty kidney are known as diseases with unremarkable urinary findings.

\section{Notable points in adult CKD}

- Because many adult patients develop chronic glomerulonephritis, it is important to recognize urinary abnormalities.

- Many cases involve lifestyle-related CKD, so it is important to modify lifestyles by diet and daily life education. Treatment with ACE inhibitors or ARBs is considered as needed.

- A CKD patient should be referred in a timely manner to a nephrologist for further examination based on the level of proteinuria, decline rate of eGFR, and past history of health examination and laboratory tests.

\section{Prevailing kidney diseases in adults (Table 12-1)}

1. Primary kidney diseases predominating in adults

- The most prevalent cause of kidney dysfunction in young adults is chronic glomerulonephritis. IgA nephropathy ranks first as a chronic glomerulonephritis leading to ESKD. The frequency of membranous nephropathy increases after middle age. Attention should be paid to the association of malignancy with membranous nephropathy.

2. Secondary kidney diseases predominating in adults

- Diabetic nephropathy has become the most frequent secondary disease as well as causative disease for dialysis induction in recent years (Fig. 12-1). In addition, obesity- and lifestyle-related kidney diseases are to be recognized.

- Diabetic nephropathy is suspected when there is a 5 -year or longer history of diabetes, persisting urinary protein excretion of $0.5 \mathrm{~g} /$ day or more, and presence of diabetic retinopathy.

*In adults, physicians consider metabolic syndromes including obesity, hypertension, dyslipidemia, and glucose intolerance.

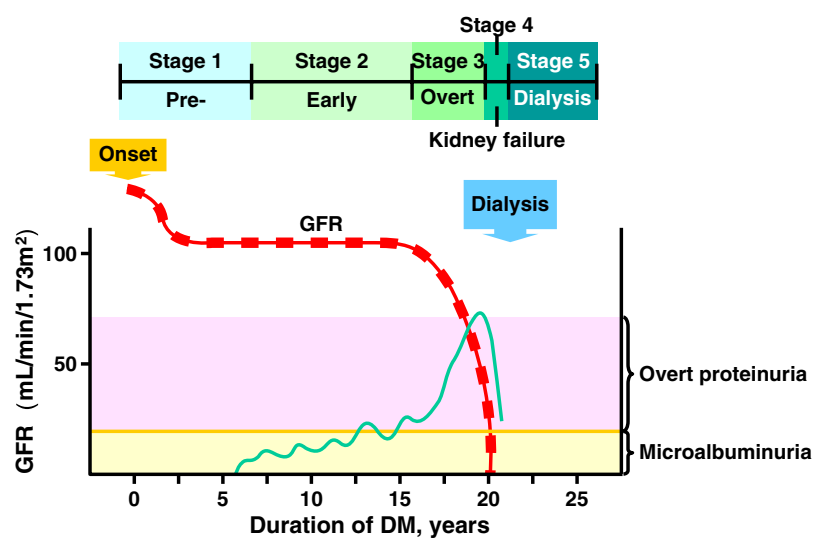

Fig. 12-1 Clinical course of type 2 diabetic nephropathy 
Table 12-1 Common kidney diseases in adults

\begin{tabular}{llll}
\hline & Primary & Secondary & Hereditary/congenital \\
\hline Glomerular disease & IgA nephropathy & Diabetic nephropathy & Alport syndrome \\
& Minimal change nephrotic syndrome & Hypertensive nephropathy (nephrosclerosis) & Fabry disease \\
& $\begin{array}{l}\text { Focal segmental glomerulosclerosis } \\
\text { Membranous nephropathy }\end{array}$ & Lupus nephritis & Benign familial hematuria \\
& Membranoproliferativeglomerulonephritis & Hepatitis C-associated nephropathy & \\
& Primary crescentic glomerulonephritis & & Polycystic kidney disease \\
$\begin{array}{l}\text { Tubulo-interstitial } \\
\text { and urinary tract } \\
\text { disease }\end{array}$ & Chronic interstitial nephritis & Gouty kidney & Ischemic nephropathy \\
\hline
\end{tabular}

